\title{
Non-Covalent Microgel Particles Containing Functional Payloads: Coacervation of PEG-Based Triblocks via Microfluidics
}

Cynthia X. Wang ${ }^{\dagger}$, Stefanie Utech ${ }^{\ddagger}$, Jeffrey D. Gopez ${ }^{\dagger}$, Mathijs F. J. Mabesoone ${ }^{\S}$, Craig J. Hawker $^{* \dagger}$, Daniel Klinger ${ }^{* \dagger}$

† Materials Research Laboratory, University of California, Santa Barbara, California 93106, United States

‡ Institute of Particle Technology, Friedrich-Alexander University, Erlangen-Nürnberg, Haberstr. 9a, 91058 Erlangen, Germany

$\diamond$ Institut für Pharmazie, Freie Universität Berlin, Königin-Luise-Str. 2-4, 14195 Berlin, Germany

$\S$ Institute for Molecules and Materials, Radboud University Nijmegen, Heyendaalseweg 135, 6525 Nijmegen, The Netherlands

\section{Corresponding Authors}

*hawker@mrl.ucsb.edu

*daniel.klinger@fu-berlin.de 


\section{A. Synthesis of triblock copolymers as microgel precursors}
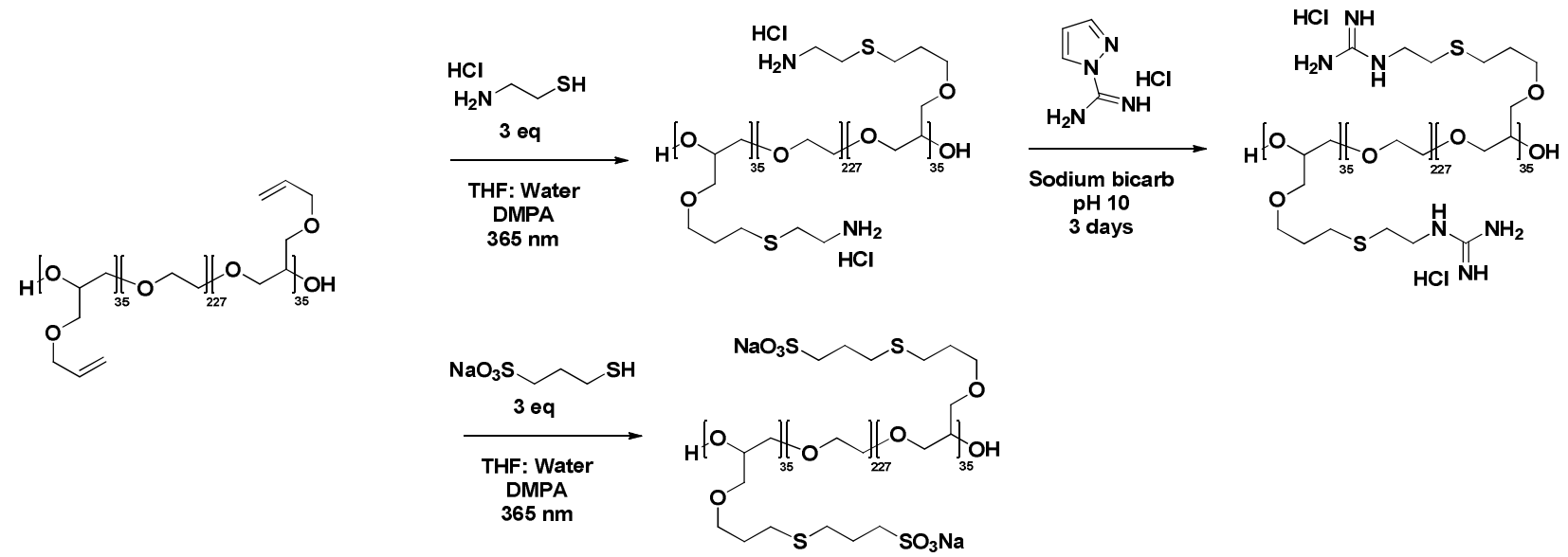

Scheme S1: Post functionalization of PAGE-PEO-PAGE to achieve ionic end-blocks (G-PEO-G and S-PEO-S) via thiolene chemistry. Synthetic procedure based on literature reference. ${ }^{22}$

$\mathrm{P}(\mathrm{AGE})_{35}-b-\mathrm{P}(\mathrm{EO})_{227}-b-\mathrm{P}(\mathrm{AGE})_{35}$ triblock copolymer was prepared by anionic ring-opening polymerization of allyl glycidyl ether (AGE) from a 1okDa PEO diol macroinitiator as previously reported. ${ }^{22} \mathrm{M}_{\mathrm{n}}$ estimated by GPC based on polystyrene calibrations: 18 kDa, PDI: 1.1. Synthetic procedure shown in $\mathrm{S}$.

${ }^{1} \mathrm{H}-\mathrm{NMR}$ in $\mathrm{D}_{2} \mathrm{O}$ solvent of $\mathrm{P}(\mathrm{Guan})_{35}-b-\mathrm{P}(\mathrm{EO})_{227}-b-\mathrm{P}(\text { Guan })_{35}$ triblock copolymer $\left(500 \mathrm{MHz}, \mathrm{D}_{2} \mathrm{O}\right.$ reference set to $\left.\delta_{\mathrm{H}}: 4 \cdot 79 \mathrm{ppm}\right)$ $\delta_{\mathrm{H}}$ : copolymer $1.91(\mathrm{~m}, 2 \mathrm{H}), 2.68(\mathrm{t}, 2 \mathrm{H}), 2.81(\mathrm{t}, 2 \mathrm{H}), 3.44(\mathrm{t}, 2 \mathrm{H}), 3.6-3.8(\mathrm{br} \mathrm{m}$, backbone and pendant ether groups) ppm.

${ }^{1} \mathrm{H}-\mathrm{NMR}$ in $\mathrm{D}_{2} \mathrm{O}$ solvent of $\mathrm{P}(\text { Sulf })_{35}-\mathrm{b}-\mathrm{P}(\mathrm{EO})_{227}-\mathrm{b}-\mathrm{P}(\text { Sulf })_{35}$ triblock copolymer: $\left(500 \mathrm{MHz}, \mathrm{D}_{2} \mathrm{O}\right.$ reference set to $\left.\boldsymbol{\delta}_{\mathrm{H}}: 4.79 \mathrm{ppm}\right) \boldsymbol{\delta}_{\mathrm{H}}$ : $1.95(\mathrm{~m}, 2 \mathrm{H}), 2.09(\mathrm{~m}, 2 \mathrm{H}), 2.73(\mathrm{t}, 2 \mathrm{H}), 2.78(\mathrm{t}, 2 \mathrm{H}), 3.09(\mathrm{t}, 2 \mathrm{H}), 3.6-3.8(\mathrm{br} \mathrm{m}$, backbone and pendant ether groups) ppm. 


\section{B. Time-dependent transfer from oil into buffer}

Microgels were prepared from aqueous solutions of guanidinium triblock copolymer (G-PEO-G) and sulfonate triblock copolymer (S-PEO-S). These solutions (referred to as polymer feed) were mixed in the microfluidic flow-focusing device in Fig. S1.

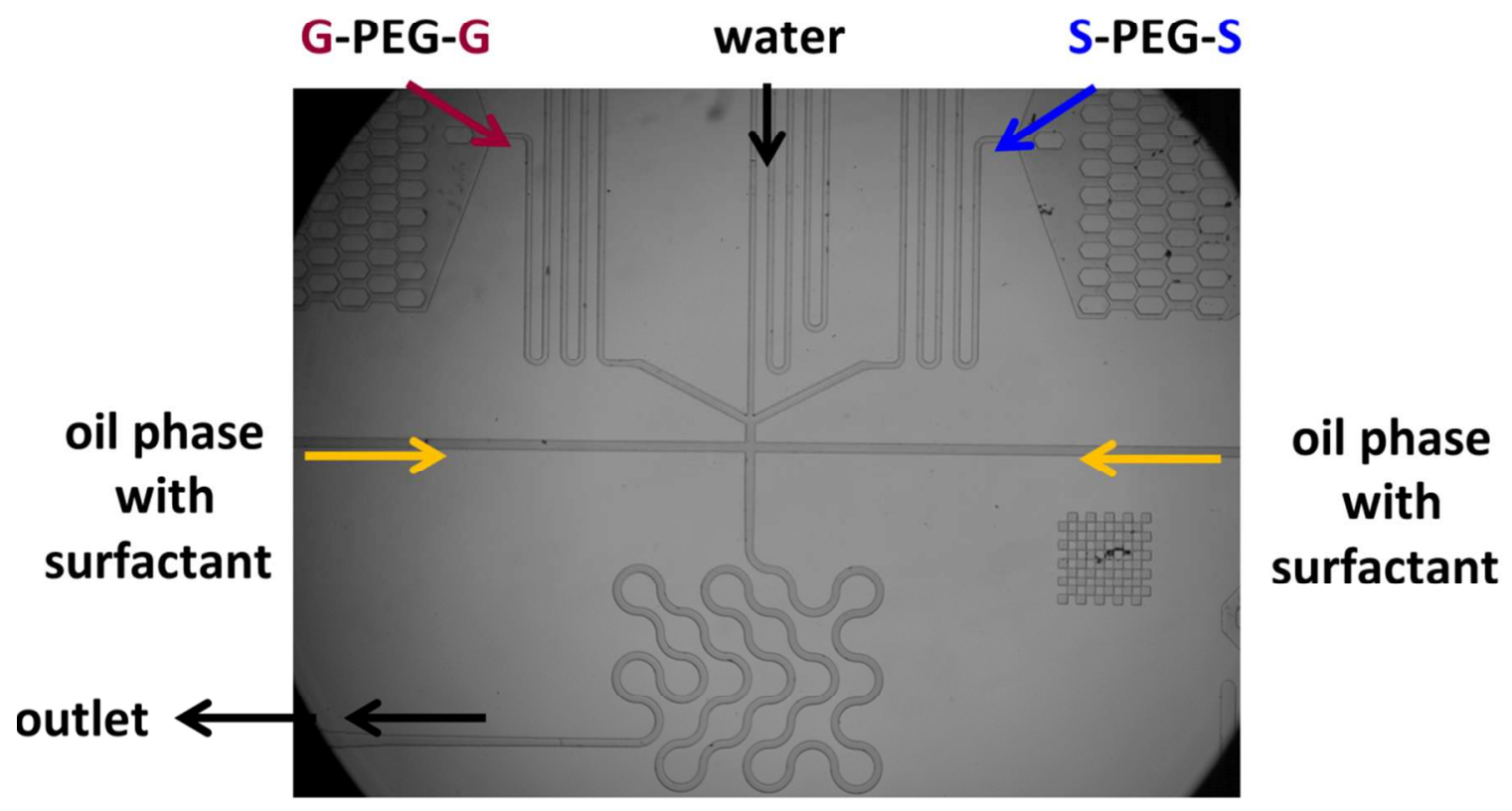

Figure S1. Optical image of droplet generator device with $50 \mu \mathrm{m}$ channels.

The 5,10 and 20 wt \% feed corresponds to the wt \% of guanidinium triblock copolymer. Due to the slightly different molecular weight of the sulfonate functionalities, an equimolar amount of sulfonate triblock copolymer feed corresponds to 5.28 wt \%, $10.55 \mathrm{wt} \%, 21.1 \mathrm{wt} \%$. The eluted microgels were collected in a vial and aliquots were removed for optical analysis. To determine whether stable microgels required longer equilibration time within the droplet, time dependent transfer of the gels from oil to buffer was carried out. No significant change was observed in the morphology of the microgels aged in buffer beyond 1 day (see Figure S2). 

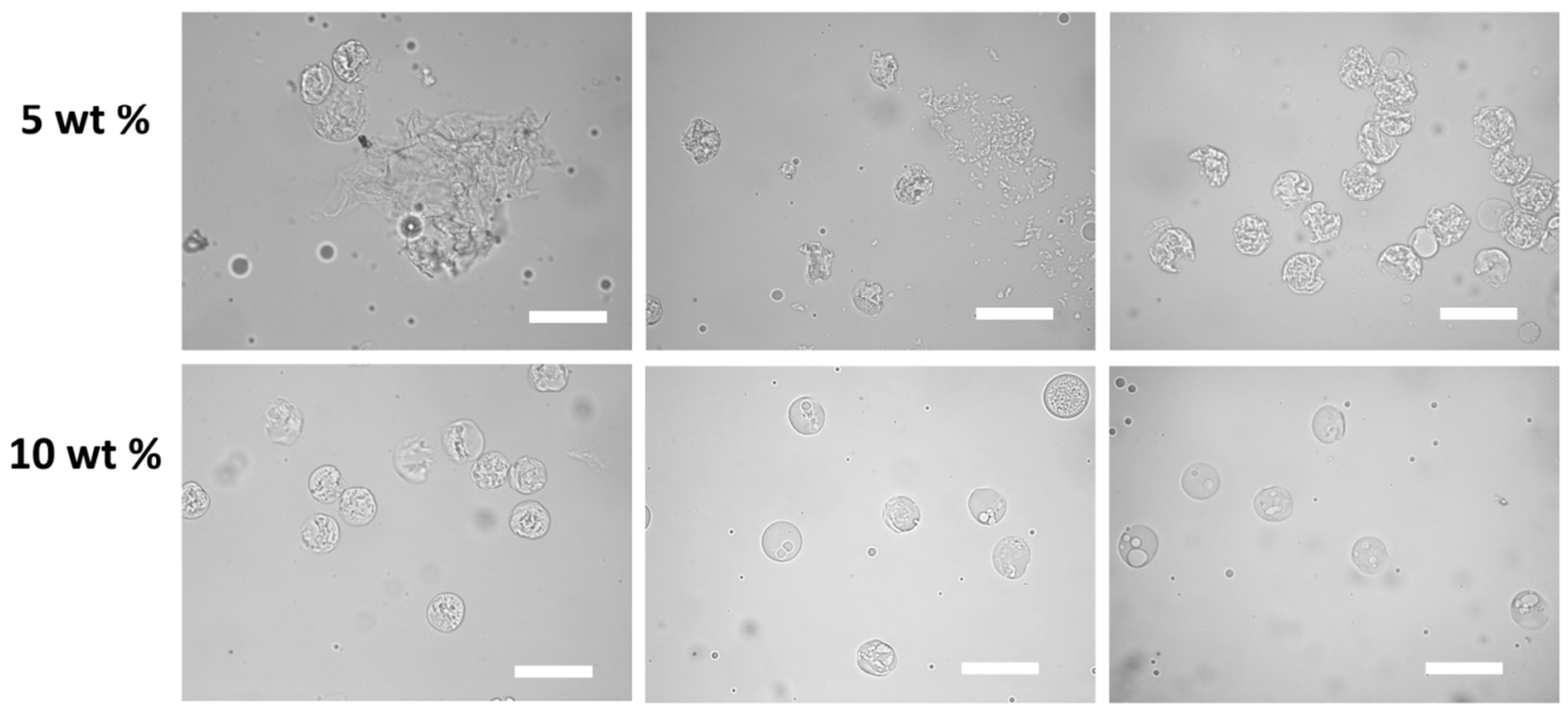

Immediate

1 day

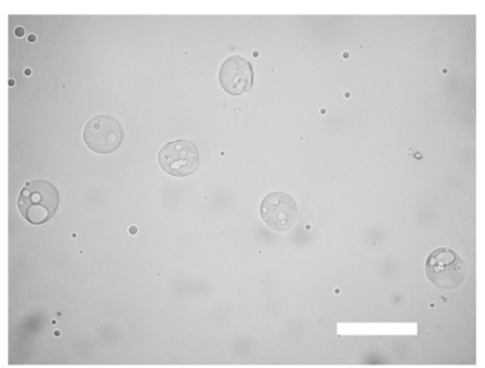

5 days

Figure S2. Microgels are stable in buffer when aged for at least 1 day in oil prior to transfer. Scale bars are $100 \mu \mathrm{m}$. Note: The smaller, darker particles visible alongside the microgels are likely debris from the glass slide or oil droplets resulting from the transfer to aqueous medium. 


\section{Microgels as prepared from a microfluidic device}

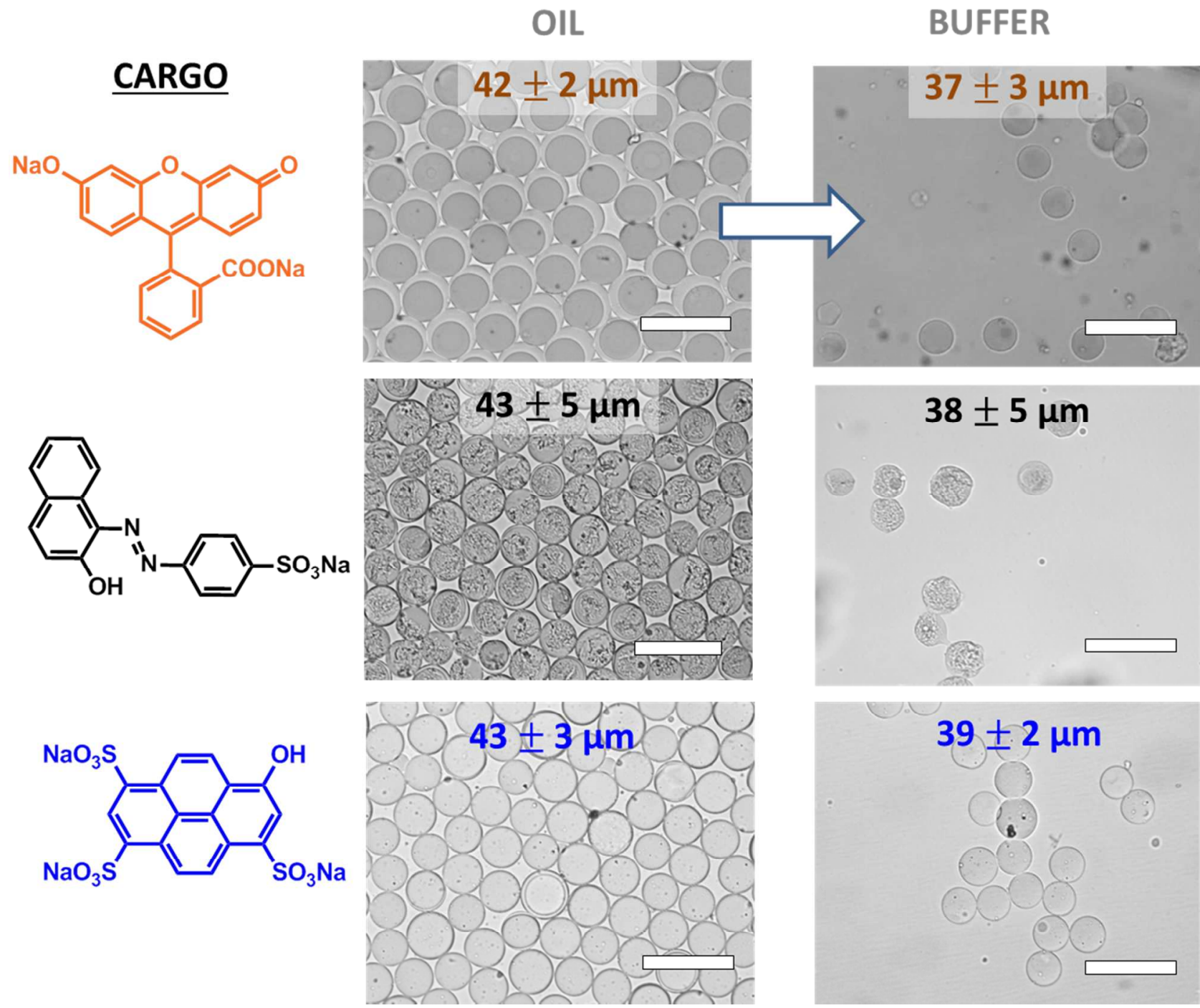

Figure S3: Optical micrographs of gels: as eluted from the device in the oil phase and after transfer to PBS ( $\mathrm{pH} 7.4,10 \mathrm{mM}$ ) using the fluorinated alcohol as transfer surfactant to stabilize the droplets in water. The cargo incorporated into the microgels is indicated on the left (fluorescein carboxylate, orange II or pyranine). For microgels loaded with fluorescein, partial droplet coalescence is most likely an artefact from the high local concentration of microgels on the glass slide during microscopy. No macroscopic aggregation was visually observed in the dispersions. For the microgels loaded with orange II, small inhomogeneities in the hydrogel texture can be observed. It is assumed that the lower solubility of the dye further slows down the network rearrangement during the aging process. This might lead to slight inhomogenities in the final particles. Scale bars are $100 \mu \mathrm{m}$. 

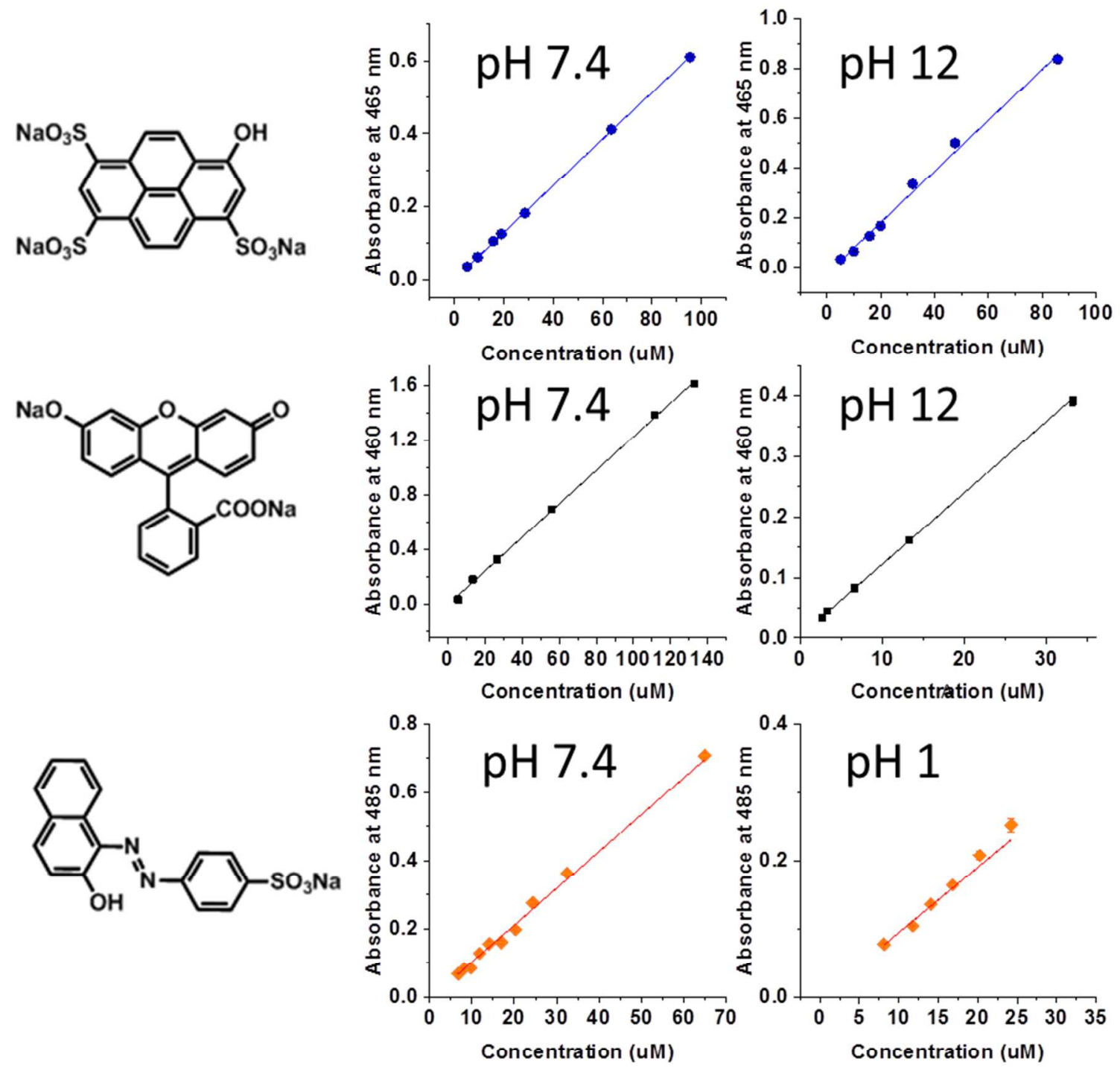

Figure S4. Calibration curves used to determine amount of dye in supernatant and in degraded microgels. The solutions for $\mathrm{pH} 12$ were prepared by the addition of $2 \mathrm{M} \mathrm{NaOH}$ to adjust the $\mathrm{pH}$ of fluorescein or pyranine stock solutions and the solutions for $\mathrm{pH} 1$, by addition of $2 \mathrm{M} \mathrm{HCl}$ to adjust the $\mathrm{pH}$ of orange II solutions. All samples were prepared in triplicate and the average absorbance plotted against concentration. 


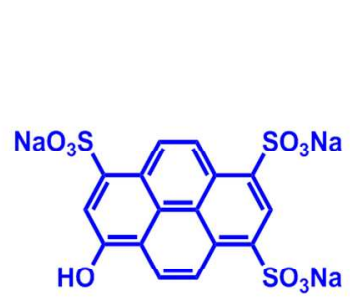
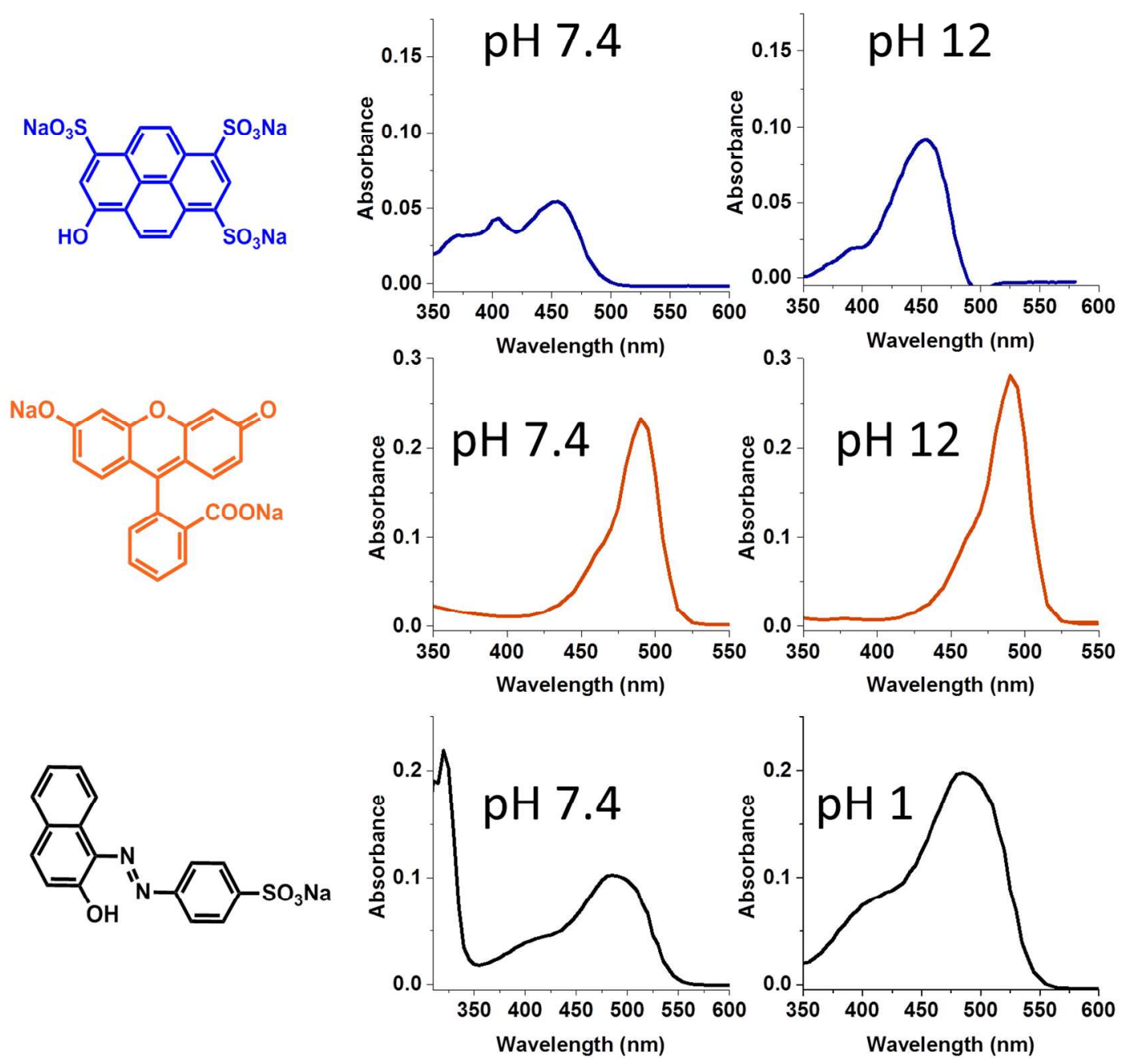

Figure S5. UV-Vis absorbance spectra of dyes from supernatant and disintegrated microgels. The left column exemplary shows spectra from the supernatant in the release experiments at $t=9 h$. The right column depicts spectra from the disintegrated microgels. The solutions for $\mathrm{pH} 12$ were prepared by the addition of $2 \mathrm{M} \mathrm{NaOH}$ to disintegrate fluorescein or pyranine loaded microgels and the solutions for $\mathrm{pH} 1$, by addition of $2 \mathrm{M} \mathrm{HCl}$ to disintegrate orange II loaded microgels. 


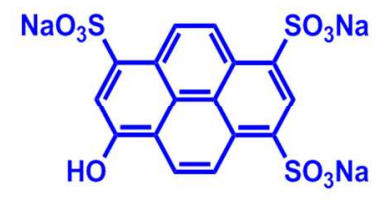

pH 7.4
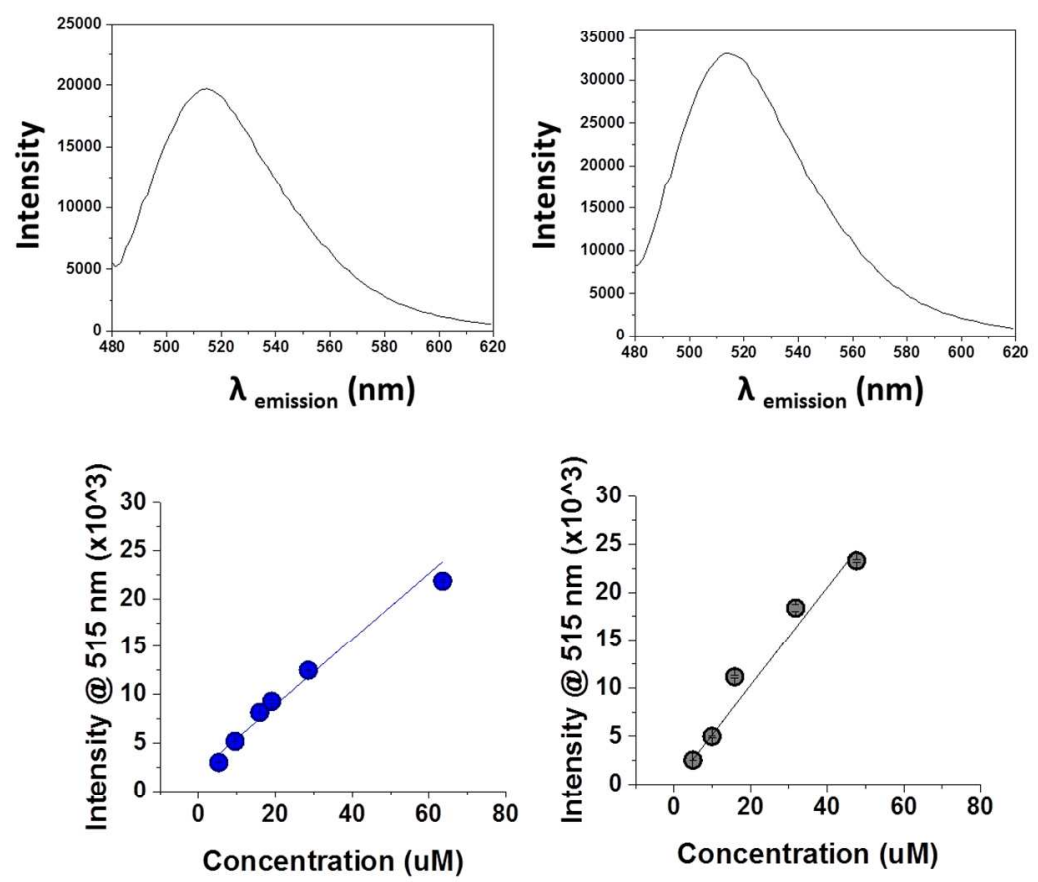

Figure S6. Fluorescence emission of pyranine and calibration curves. 